\title{
Extraskeletal Myxoid Chondrosarcoma-like Features in Metastasizing Lung Adenocarcinoma: Unique Morphological Changes after Chemo-radiotherapy
}

Susumu Matsukuma ${ }^{1,2 *}$, Aoi Yamashiro1, Kimi Kato1, Hiroaki Takeo' and Kimiya Sato ${ }^{1}$

${ }^{1}$ Department of Pathology, Japan Self Defense Forces Central Hospital, Tokyo, Japan

${ }^{2}$ Health Care Center, Japan Self Defense Forces Central Hospital, Tokyo, Japan

\begin{abstract}
Lung cancer closely resembling Extraskeletal Myxoid Chondrosarcoma (EMC), called "primary pulmonary myxoid sarcoma (PPMS)," is an extremely rare tumor. This report is presented here of a unique case showing EMC-like changes only in metastases from conventional lung adenocarcinoma in an 81-year-old man. After lung tumor resection and chemo-radiotherapy, he underwent the resection of right adrenal metastasis composed of carcinomatous and EMC-like components. There were multifocal transitions between these components. He was treated with additional chemo-radiotherapy for newly developed liver and brain metastases, but he died 3.3 years after the initial surgery. Autopsy revealed EMC-like tumors, with or without minute adenocarcinomatous components, extensively involving widespread organs. Reverse transcription-polymerase chain reaction did not detect EWSR1CREB1 fusion in the primary tumor or metastases. These features indicated that the current tumor was different from PPMS characterized by EWSR1-CREB1 fusion. We concluded that such EMC-like features represent morphological changes of metastatic lung adenocarcinoma. We believe that this possible occurrence should be recognized for accurate diagnosis of metastatic lung carcinoma.
\end{abstract}

Keywords: Chemo-radiotherapy; Extraskeletal Myxoid Chondrosarcoma; EWSR1-CREB1; Lung; Adenocarcinoma

\section{Introduction}

Extraskeletal Myxoid Chondrosarcoma (EMC) is an uncommon soft-tissue tumor usually originating in the deep tissues [1]. Primary lung cancers resembling EMC have been rarely reported as "primary pulmonary myxoid sarcoma (PPMS)" [2,3]. Recently, we encountered a unique autopsy case of lung carcinoma showing EMC-like features only in metastases after chemo-radiotherapy. To our knowledge, such EMC-like changes have not been mentioned previously in metastases of lung carcinoma, although therapy-induced myxomatous or colloid-like changes have been reported in some other site cancers [4,5]. We herein described this case to expand our knowledge of morphological changes in metastasizing lung carcinoma.

\section{Case Report}

An 81-year-old man underwent the left upper lobectomy for a $2 \mathrm{~cm}$ sized lung tumor. Before the lobectomy, the whole-body examination using X-ray computed tomography (CT) scan and bone scintiscan could not reveal any metastases, and the removed tumor was classified as Stage IA (pT1N0cM0). The patient did not receive additional chemoradiotherapy, and was followed up with laboratory examination of serum tumor markers and chest radiologic examination. About 1.5 years later, abdominal X-ray CT scan was performed for his progressive weight loss in a year, and revealed a right adrenal metastasis. He was treated with local radiotherapy and chemotherapy using gemcitabine, vinorelbine, and tegafur. Because of its progressive increase 1.2 years after the beginning of chemo-radiotherapy, the right adrenalectomy was performed. Thereafter, for newly developed liver and brain metastases, he received additional chemotherapy using gemcitabine, vinorelbine, and paclitaxel, and radiotherapy for brain metastasis. However, he died 3.3 years after the initial surgery.

The well-demarcated primary lung tumor showed features of poorly differentiated adenocarcinoma (histological grade 3 ) composed of nested polygonal cells occasionally showing papillary or mucinpositive glandular features (Figure 1). Sarcomatous features were not identified. The surgically removed right adrenal metastasis included adenocarcinomatous components and myxoid areas (Figure 2a).
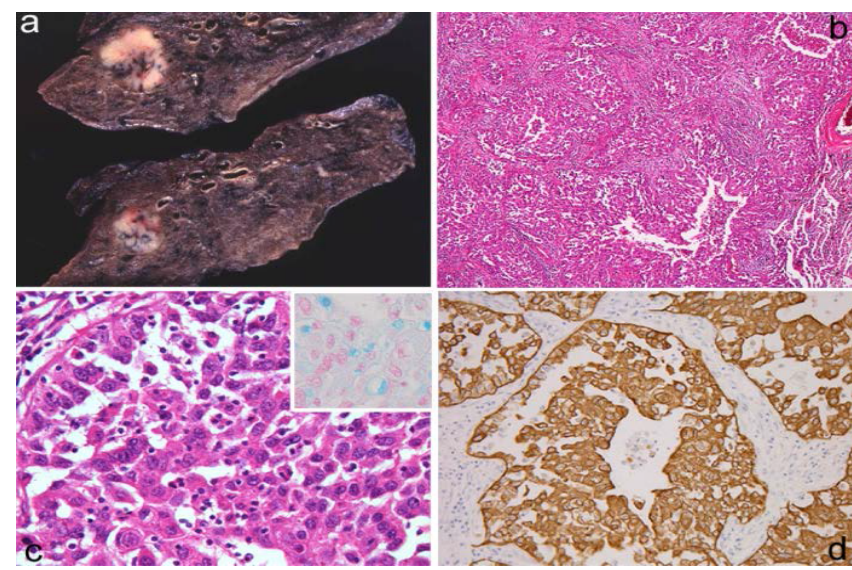

Figure 1: Primary lung adenocarcinoma (a) showing a well demarcated nodule. Low-power view (b) showing nested and vague papillary cancerous growth and high-power view (c) demonstrating polygonal cancer cells (c) with alcian blue+ lumina (c, inset). Primary carcinoma cells expressing strong positivity for cytokeratin 7 (d).

*Corresponding author: Susumu Matsukuma, Health Care Center, Japan Self Defense Forces Central Hospital, Ikejiri 1-2-24, Setagaya-ku, 154-0001, Tokyo, Japan, Tel: +81-3-3411-0151, ext 6446; Fax: +81-3-3418-0197; E-mail: skuma@cocoa.plala.or.jp

Received December 09, 2013; Accepted February 18, 2014; Published February 20, 2014

Citation: Matsukuma S, Yamashiro A, Kato K, Takeo H, Sato K (2014) Extraskeletal Myxoid Chondrosarcoma-like Features in Metastasizing Lung Adenocarcinoma: Unique Morphological Changes after Chemo-radiotherapy. J Cytol Histol 5: 220. doi:10.4172/2157-7099.1000220

Copyright: (c) 2014 Matsukuma S, et al. This is an open-access article distributed under the terms of the Creative Commons Attribution License, which permits unrestricted use, distribution, and reproduction in any medium, provided the original author and source are credited. 


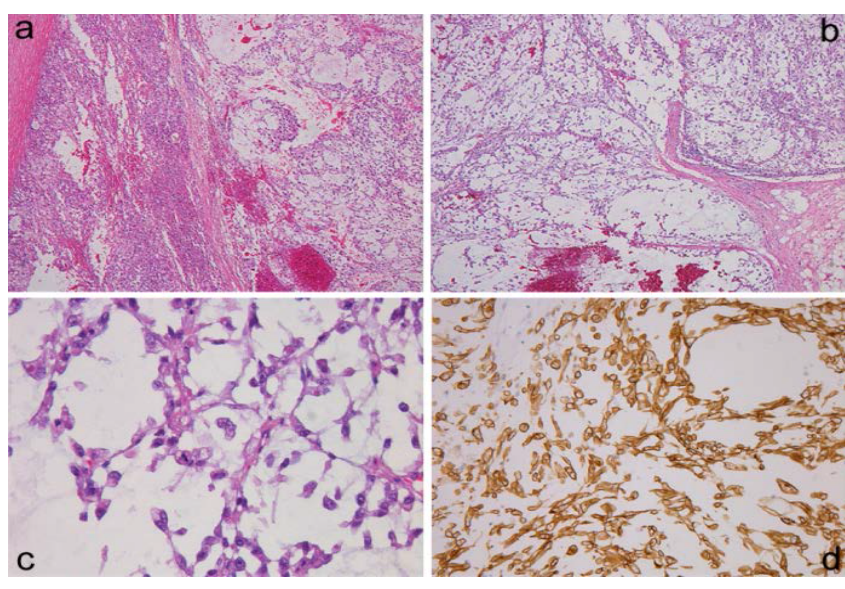

Figure 2: Surgically removed right adrenal metastasis (a) showing transition between left-sided adenocarcinomatous components and right-sided markedly myxoid area. Low-power view of postmortem lymph node metastasis (b) composed of lobulated myxoid areas containing reticular cancerous proliferation, and the high-power view (c) showing polygonal or spindle cells arranged in short anastomosing cords with an abundant myxoid material, mimicking extraskeletal myxoid chondrosarcoma. Sarcomatous spindle cells in the liver metastasis showing cytokeratin 7 expression (d).

The latter consisted of faintly eosinophilic spindle or polygonal cells proliferating in a fascicular or reticular fashion, mimicking EMC. There were multifocal transitions between these carcinomatous and sarcomatous components. Autopsy revealed EMC-like tumors extensively involving both lungs, liver, peritoneum, left adrenal gland, brain, and generalized lymph nodes (Figures $2 \mathrm{c}$ and $2 \mathrm{~d}$ ). Minute concomitant adenocarcinomatous features were found focally in these myxoid tumors.

Adenocarcinomatous components in both the primary tumor and metastases were diffusely positive for pan-cytokeratin (pan-CK), CK7 (Figure 1d), epithelial membrane antigen (EMA), focally positive for carcinoembryonic antigen (CEA), Napsin A, and vimentin, but were negative for CK20, thyroid transcription factor-1 (TTF-1), S-100 protein, CD34, desmin, smooth muscle actin (SMA), or HMB45. EMC-like components in both the right adrenal metastasis and postmortem metastases were focally positive for pan-CK, CK7 (Figure 2d), EMA, and vimentin, but were negative for CEA, Napsin A, TTF-1, SMA, S-100 protein, or CD34. Reverse transcription-polymerase chain reaction (RT-PCR) using the formalin-fixed, paraffin-embedded tissues (the primers and PCR conditions used were described elsewhere [6]) did not detect EWSR1-CREB1 and EWSR1-ATF1 fusions in the primary tumor, surgically removed right adrenal metastasis, or postmortem liver myxoid tumor.

\section{Discussion}

In this case, there were distinct transitions between adenocarcinomatous components and EMC-like features chiefly in right adrenal metastasis and focally in postmortem specimens. These findings suggested that EMC-like features represent secondary changes of pulmonary adenocarcinoma. In addition, in the primary lung tumor before chemo-radiotherapy, EMC-like features were not identified. These findings raised a possibility that such therapy may play a role in EMC-like changes of metastases. Previous reports have described therapy-induced histological alterations, such as necro-inflammatory response in non-small lung carcinoma [7], massive myxomatous response in prostatic and rectal adenocarcinomas [4,5], clear cell carcinoma-like features in ovarian carcinoma [8], and granulosa cell tumor-like pattern in ovarian sex cord tumor with annular tubules [9]. However, therapy-induced EMC-like changes have not been mentioned previously in lung or other site carcinoma. Moreover, in the current case, the histology of metastatic sites before chemoradiotherapy was unknown. Widespread metastatic lesions without radiotherapy also showed EMC-like changes. Hence, the current study could not determine whether EMC-like changes in this case were a chemo-radiotherapy-induced condition or a simply manifestation of metastases themselves.

Primary lung tumors resembling EMC include PPMS [2,3], pulmonary angiomatoid fibrous histiocytoma (AFH) [10], and pulmonary microcysticfibromyxoma [11]. PPMS is a rare tumor of uncertain differentiation, characterized by EMC-like histology and EWSR1-CREB1 fusion [2,3], and this entity would encompass "lowgrade pulmonary myxoid sarcoma" [12] and "primary pulmonary EMC" [13]. The currently presented primary and secondary tumors showed pan-CK/CK7+ and no EWSR1-CREB1 fusion, which could rule out a diagnosis of PPMS. Similarly, $\mathrm{CK}+$ in the current case is distinctively different from no CK expression in pulmonary $\mathrm{AFH}$ and microcysticfibromyxoma [10,11]. No EWSR1-ATF1 fusion in the present case can rule out a diagnosis of AFH [10]. Lung myoepithelioma with extensive myxoid changes may be another differential diagnosis of this case. However, most cases of myoepithelioma were S-100 protein+ and/or SMA+ $[3,14]$, whereas such expressions were not observed in the current case. Hence, the present case would represent no myoepithelial differentiation in primary or secondary lesions.

Thus, EMC-like changes can occur in metastasizing lung adenocarcinoma. Consideration of this possible occurrence would be useful for accurate diagnosis and therapeutic management of patients with lung carcinoma, although it may be a rare phenomenon.

\section{Acknowledgement}

We thank Dr. Masonori Hisaoka, Department of Pathology and Oncology, School of Medicine, University of Occupational and Environmental Health Kitakyushu, Japan, for reverse transcription-polymerase chain reaction examination of this case.

\section{References}

1. Weiss SW, Goldblum JR (2008) Enzinger \& Weiss's soft tissue tumors. (5thedn), Mosby/Elsevier, Philadelphia, PA, USA.

2. Thway K, Nicholson AG, Lawson K, Gonzalez D, Rice A, et al. (2011) Primary pulmonary myxoid sarcoma with EWSR1-CREB1 fusion: a new tumor entity. Am J SurgPathol 35: 1722-1732.

3. Matsukuma S, Hisaoka M, Obara K, Kono T, Takeo H, et al. (2012) Primary pulmonary myxoid sarcoma with EWSR1-CREB1 fusion, resembling extraskeletal myxoid chondrosarcoma: Case report with a review of Literature. Pathollnt 62: 817-822.

4. Tran TA, Jennings TA, Ross JS, Nazeer T (1998) Pseudomyxomaovariilikeposttherapeutic alteration in prostatic adenocarcinoma. A distinctive pattern in patients receiving neoadjuvant androgen ablation therapy. Am J SurgPathol 22: 347-354.

5. Rullier A, Laurent C, Vendrely V, Le Bail B, Bioulac-Sage P, et al. (2005) Impact of colloid response on survival after preoperative radiotherapy in locally advanced rectal carcinoma. Am J SurgPathol 29: 602-606.

6. Hisaoka M, Ishida T, Kuo TT, Matsuyama A, Imamura T, et al. (2008) Clear cell sarcoma of soft tissue: a clinicopathologic, immunohistochemical, and molecular analysis of 33 cases. Am J SurgPathol 32: 452-460.

7. Liu-Jarin X, Stoopler MB, Raftopoulos H, Ginsburg M, Gorenstein L, et al. (2003) Histologic assessment of non-small cell lung carcinoma after neoadjuvant therapy. Mod Pathol 16: 1102-1108. 
Citation: Matsukuma S, Yamashiro A, Kato K, Takeo H, Sato K (2014) Extraskeletal Myxoid Chondrosarcoma-like Features in Metastasizing Lung Adenocarcinoma: Unique Morphological Changes after Chemo-radiotherapy. J Cytol Histol 5: 220. doi:10.4172/2157-7099.1000220

Page 3 of 3

8. Chew I, Soslow RA, Park KJ (2009) Morphologic changes in ovarian carcinoma after neoadjuvant chemotherapy: Report of a case showing extensive clear cell changes mimicking clear cell carcinoma. Int J GynecolPathol 28: 442-446.

9. Khalifa M, Nofech-Mozes S, Atri M, Covens A (2006) Chemotherapy-induced alterations in ovarian sex cord tumor with annular tubules (SCTAT): a diagnostic challenge. Pathol Res Pract 202: 183-186.

10. Thway K, Nicholson AG, Wallace WA, Al-Nafussi A, Pilling J, et al. (2012) Endobronchial pulmonary angiomatoid fibrous histiocytoma: two cases with EWSR1-CREB1 and EWSR1-ATF1 fusions. Am J SurgPathol 36: 883-888.

11. Shilo K, Miettinen M, Travis WD, Timens W, Nogueira R, et al. (2006)
Pulmonarymicrocysticfibromyxoma: Report of 3 cases. Am J SurgPathol 30: 1432-1435.

12. Inayama Y, Hayashi H, Ogawa N, Mitsui H, Nakatani Y (2001) Low-grade pulmonary myxoid sarcoma of uncertain histogenesis. Pathollnt 51: 204-210.

13. Zhou Q, Lu G, Liu A, Kohno T (2012) Extraskeletalmyxoidchondrosarcoma in the lung: asymptomatic lung mass with severe anemia. DiagnPathol 7: 112.

14. Higashiyama M, Kodama K, Yokouchi H, Takami K, Kabuto T, et al. (1998) Myoepithelioma of the lung: report of two cases and review of the literature. Lung Cancer 20: 47-56. 